\title{
INTELIGENT CRACK DETECTING ALGORITHM ON THE CONCRETE CRACK IMAGE USING NEURAL NETWORK
}

\author{
Hyeong-Gyeong Moon and Jung-Hoon Kim* \\ Department of Civil and Environmental Engineering, Yonsei University, Seoul, Korea \\ *Corresponding author (junghoon@yonsei.ac.kr)
}

\begin{abstract}
Safety inspection of concrete structures should be strictly carried out since it is closely related with the structural health and reliability. However, it is difficult to find cracks by a visual check for the extremely large structures. So, the development of crack detecting systems has been a significant issue. Final objective of this research is to develop an automatic crack detection system that can analyze the concrete surface and visualize the cracks efficiently. The algorithm is composed of two parts; image processing and image classification. In the first step, cracks are distinguished from background image easily using the filtering, the improved subtraction method, and the morphological operation. The particular data such as the number of pixel and the ratio of the major axis to minor axis for connected pixels area are also extracted. In the second step, the existence of cracks are identified. Backpropagation neural network is used to automate the image classification. Target data values in the training process were generated by inspector's manual classification. In order to verify the first and second step of the proposed algorithm, the algorithm was tested using real surface images of concrete bridge. Backpropagation neural network was trained using 105 images of concrete structure, and the trained network was tested for new 120 new images. The recognition rate of the crack image was 90\% and non-crack image was 92\%. This method is useful for non expert inspectors, enabling them to perform crack monitoring tasks effectively.
\end{abstract}

Keywords: Automatic Concrete Crack Detection, Image processing, Construction Safety and Maintenance, Backpropagation Neural Network

\section{INTRODUCTION}

The stability of structure is an important factor not only in the construction phase but also in the maintenance phase. The importance of safety in facility is increased as the construction of high-rise buildings, super long span bridges, and asymmetric buildings are popular. For this reason, construction safety management system is actively under development these days. In addition, the interest in the automation of the construction management system has been increased, because the civil infrastructure under the management is large in scale, the regular evaluations are needed to guarantee the continuity in service, running cost is very expensive, and the safety of workers should be ensured. By developing an effective infrastructure lifecycle management system through automation, it is possible to secure the stability of the facility, and reduce the number of inspectors, inspection, time, and maintenance cost. Moreover, we can judge the condition of the structural health objectively by acquiring and processing the data.

Most of the infrastructures are composed of concrete. In these structures, the one of the ways in judging the structural health is to examine a crack on the surface of the structure. Since the condition of a concrete structure can be easily and directly identified by inspecting the surface crack, the crack assessment should be done on a regular basis to ensure durability and safety within its life-cycle. So many researchers have studied the automated concrete crack detecting method. Some researchers acquired the data from structures by using CCTV, laser scanner[1], and microwave[2]. Among them, the utilization of the image data acquisition is widely used. In this case, variety of processing methods have been proposed: image processing using edge detection technique[3], histogram 
matching, image filtering and change detection[4], and automatic thresholding valley-emphasis method-a revised version of the Otsu method for detecting small to large defects[5].

Besides the processing the image data, it is required to distinguish defects automatically to reduce the effort of the human inspectors. Sinha et al.[6] employed classification through neuro-fuzzy network and Khanfar et al.[2] detected the concrete structure defects through fuzzy logic techniques. In addition to this method, neural network and genetic algorithm have been used. In many papers, the inspection methods vary widely from data acquisition to classification and these show that diversity of algorithms can be realized. This fact implies that the construction environment is very varied. But the proposed methods on the aforementioned papers have limits in environmental condition. For example, the environment for sewer pipe[1, 4] inspection is not severely affected by weather condition. So it is difficult to apply the inspection algorithm to the structures that are exposed to various weather conditions. When the inspection algorithm is influenced by external conditions, a system engineer should participate in the inspection of structures, because parameter tuning requires knowledge of experts. To solve this problem, this paper aims to develop the algorithm that allows anybody to tune the parameter easily without a system engineer

The proposed algorithm classifies into the cracked concrete image and the non-cracked concrete image using the image processing and a neural network. The architecture of entire algorithm is shown in Fig.1. Section 2 explains the step 1, the image processing method and section 3 describes on the selection of image processing parameters using optimization technique. Section 4 describes on the step2, the classification of cracked and non-crack images. Section 5 describes the experimental result of the proposed algorithm. Conclusions and future work is explained in the final section.

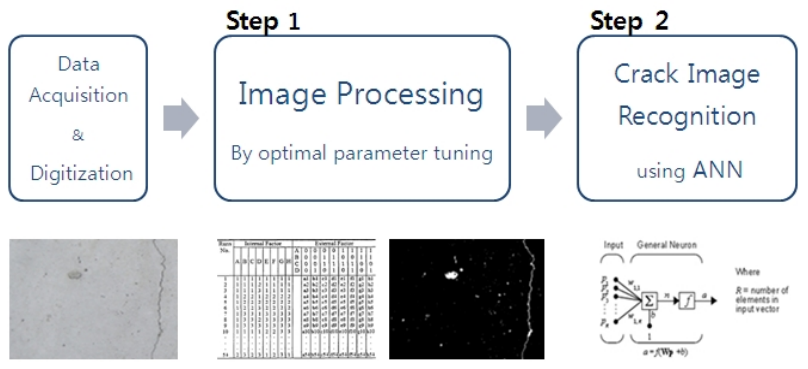

Fig. 1 Algorithm Architecture

\section{IMAGE PROCESSING}

It may be possible to classify cracked and non-cracked images directly from the original images by using Artificial Neural Network (ANN). However, the method using only ANN without image processing will require more computation time, since the training images have so many information. Thus it is required to include the image processing before applying ANN step in order to develop an effective process.

Image processing step for concrete crack detection consists of the following procedures. (1) Transformation of the acquired digital color image into a gray-level image; (2) Improved subtraction method for the image in uniform brightness; (3) Gaussian filtering for smoothing; (4) Binarization; (5) Morphological processing using closing and labeling. These five procedures are shown in Fig. 2

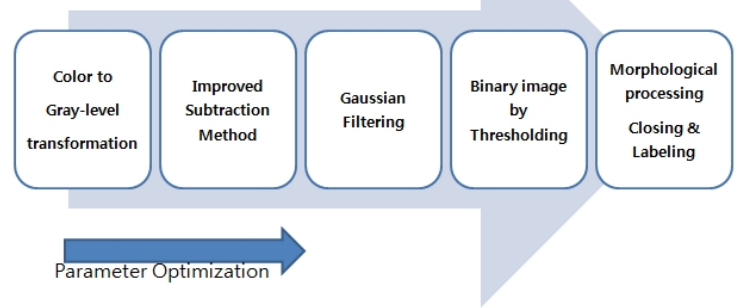

Fig. 2 Image processing step including parameter optimization architecture

\subsection{Color to Gray level transformation}

This step converts the true-color image RGB to the graylevel intensity image by eliminating the hue and saturation information while retaining the luminance[7]. In this process, the amount of image data decreases. For example, the RGB color pixel having a depth of 24 bit 
$\left(\left(2^{8}\right)^{3}=16,777,216\right.$ color) is converted into 8 bit gray-level ( $2^{8}=256$ level).

\subsection{Improved Subtraction Processing}

Gray-level image obtained by section 2.1 is processed with improved subtraction processing. Subtraction processing[8] generally removes the slight variation like shadings or irregularly illuminated condition. It is used in the processing of the noisy concrete surface for crack visualization. Fig. 3 shows a removal of serious variations like shading. First, a smoothed image is generated by applying a median filter on a gray-level image. Then the gray-level image is subtracted from the smoothed image. The subtracted image is obtained by the following equation[8].

$$
I_{S}\left(x_{i}\right)=\max \left\{\begin{array}{c}
\text { median }_{x_{j} \in R_{j}} I\left(x_{j}\right)-I\left(x_{i}\right) \\
0
\end{array}\right.
$$

where $I\left(x_{i}\right)$ means the intensity of the pixel $x_{i}$, and $R_{i}$ means the neighborhood of the pixel $x_{i}$ [8]. When the result of the subtraction is negative number, the result is represented with 0 .

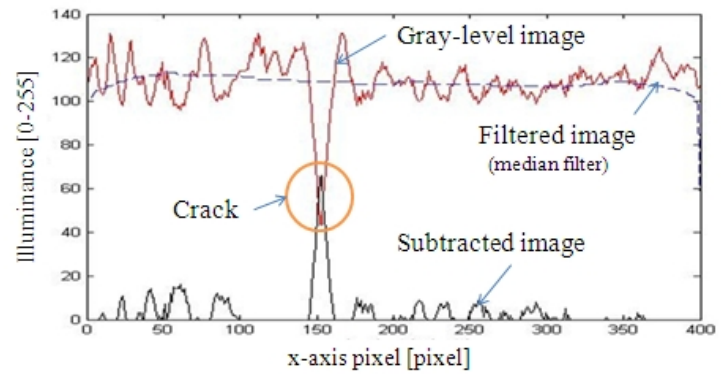

Fig. 3 Crack visualization by subtraction processing [8]

A subtracted result image could be a crack or a noisy concrete surface. In the subtraction processing, cracks can be easily identified since those will have more higher luminance than noises as shown in Fig. 3. Here, it is required to remove the noises in the original subtraction method as much as possible since it is undesirable in the next labeling processing. The labeling process will group the connected pixels into objects to identify the crack object. However, noises are also classified into objects since they are connected pixels. So this paper proposes the improved subtraction method that can precisely eliminate noises from noisy-concrete surface image. In the improved subtraction method, crack parts are highlighted by multiplying the result of the original subtraction method by some constant value in case that the result is greater than some threshold. When this thresholding is applied to the improved subtraction method, cracks can be detected effectively. The process is represented by the following equation.

$$
I_{I S}(x, y)=\left\{\begin{array}{rll}
2 \times I_{S}(x, y) & \text { if } & I_{S}(x, y)>T \\
I_{S}(x, y) & \text { if } & I_{S}(x, y) \leq T
\end{array}\right.
$$

where $I_{I S}(x, y)$ is the result of the improved subtraction method and $I_{S}(x, y)$ is the result of the original subtraction method and $T$ means the threshold. The threshold value is tuned manually. By this modification, the difference in illuminance between cracks and noises become large and it contributes on the noise reduction in the next image processing. The results of the original subtraction processing and the improved subtraction processing are compared at the end of section 2.5 .

\subsection{Gaussian Low-pass Filter}

Basically gaussian low-pass filtering smooths images. The aim of adopting this filter is to connect the small gap of the crack line. It can also adjust the distortion of the crack shape. In this process, spatial filtering that is also called as neighborhood processing [9], performs the operation with neighborhood around the center point $(\mathrm{x}, \mathrm{y})$. This process can be represented by the following equation and it repeats for all points

$$
r(x, y)=T[(o(x, y)]
$$

where $o(x, y)$ is the input image, $r(x, y)$ is the processed image, and $T$ is an operator on $o$ defined over some neighborhood of $(x, y)$. Spatial filter is can be classified into linear and nonlinear spatial filtering. In this step, the gaussian type of liner spatial filtering is used.

\subsection{Creation of binary image by thresholding}

Thresholding is the simple and intuitive method in image segmentation. Object pixels can be simply extracted from background by thresholding. This step converts an input gray-level image to a binary image where the values of points are expressed by 0 (black) or 1 (white). If the pixel values in an input image are greater than some threshold value $T$, the output pixel values are assigned into 1 and the points are called as object points. When the input pixel values are smaller than $T$, the output values are assigned 
into 0 and they are regarded as background points. The following equation represent this thresholding.

$$
r(x, y)= \begin{cases}1 & \text { if } o(x, y)>T \\ 0 & \text { if } \quad o(x, y) \leq T\end{cases}
$$

where $r(x, y)$ is thresholded image, $o(x, y)$ is gray level image. Both cracks and non-negligible noises can be assigned to the value 1 . In our process, threshold value can be determined by one of the methods among basic global thresholding, otsu method, and valley-emphasis method.

\subsection{Morphological Image Processing}

Morphological image processing can be used to extract image components such as the shape of cracks[9]. There are dilation, erosion, opening, closing, labeling, and so on in morphological operation. In the proposed algorithm, closing and labeling are used.

\section{(1) Closing}

Closing is generally used to connect narrow breaks and long thin gulfs, eliminate small holes, and fill gaps in the crack.

\section{(2) Labeling}

In this step, neighboring connected components are recognized as a single object and all the points in the single object are labeled with the same number as shown in Fig. 4. That is to say, each object will have its own label number and it is composed of connected pixels. Here, an object could be a crack object or a noise object. However, two kinds of objects will have a distinction in the area and the ratio of the major to minor axis for the area.

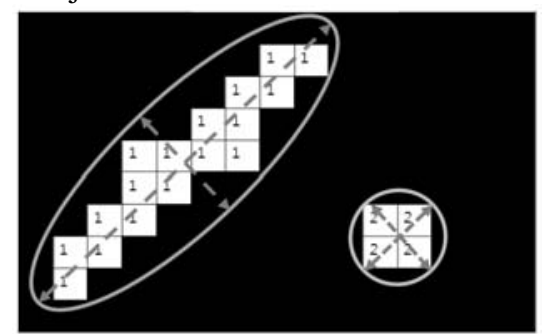

Fig. 4 Labeling and the ratio of the major axis to minor axis for connected pixels area.

Fig. 5 shows the result of the whole image processing step from gray-level transformation to morphological processing described in Fig.2. Fig. 6 shows the results of the previous subtraction processing and improved subtraction processing; (a) is original image, (b) is the result of the original subtraction processing, and (c) is the result of the whole image processing using (b).

(a)

(b)

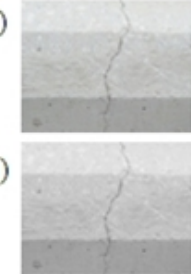

Color to Gray-leve transformation

(c)

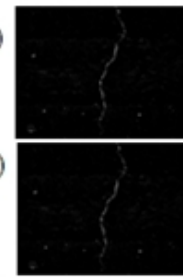

(e)
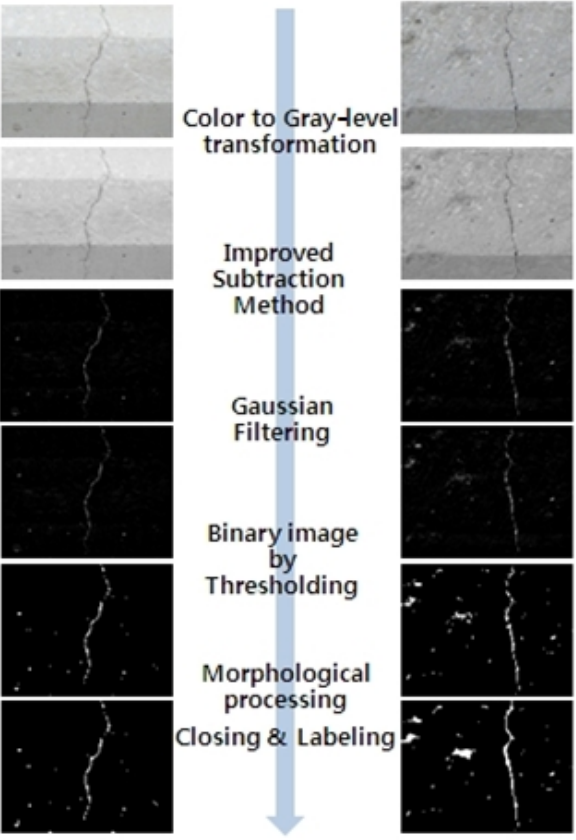

Fig. 5 Result of the proposed image processing

On the right side of Fig. 6, (d) is the result of the improved subtraction processing, and (e) is the end result of the total image processing including the improved subtraction. From this figure, we can see that the number of the noise objects in (e) is relatively small compared with (c). In addition, crack lines are clearly distinguished from the image by using the improved subtraction processing, and its effectiveness is shown.

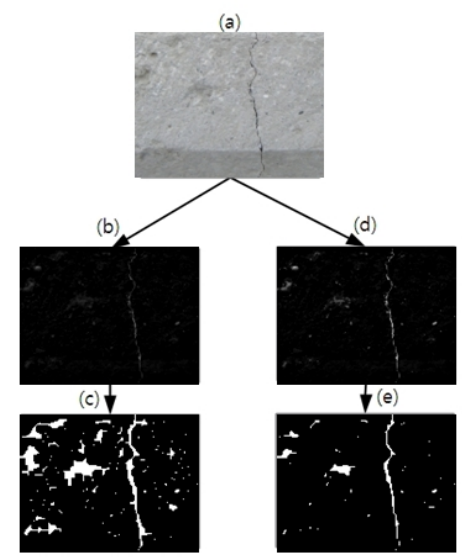

Fig. 6 Comparison of the existing and improved subtraction processing

When all steps of the image processing are completed, crack objects can be distinguished from a background 
easily. In addition, the area (total number of pixels) and the ratio of the major axis to minor axis for connected pixels area can be calculated from objects of image. By minimizing the number and the area of the noise objects, we can also optimally tune the parameters that are required in the process of section 2 . When the object has the many pixels and the greater ratio of the major axis to minor axis, there is a strong possibility that it is a crack object.

\section{PARAMETERS OPTIMIZATION}

The image processing step in section 2 requires proper selection of many parameters such as median filter size, threshold values, gaussian filter size, and the standard deviation of gaussian curve. Tuning of these parameters is not easy for the non-expert. When these parameters are optimally selected, the crack can be effectively distinguished from the background. Here, we propose to use the Taguchi method that can obtain the optimal parameters easily without help of expert. Taguchi method is experimental design optimization method which provide input parameters for optimizing the output process performance through an organized set of experiments by using orthogonal arrays.[10] It provides an efficient and systematic way to determine optimal control parameters with a minimum experimental runs. Taguchi method finds optimal level of a controllable factor based on level with the highest Signal to Noise ratio. In our application, controllable factors under optimization are median filter size, threshold value of the improved subtraction method, thresholding method, gaussian filter size, and standard deviation in gaussian filter. Each controllable factor is set to have three levels. The corresponding numerical values for the three levels of each parameter are given as shown in Table 1. The selected orthogonal array is $L_{27}$, the experiment is executed for 5 iterations. When the environmental conditions in the inspection site are changed too much, the performance of the optimization can be increased by increasing the number of iteration of experiment. In the optimization, the "larger the better" loss function is used. The parameters required in the image processing step are selected by the optimal parameters that is determined by the Taguchi method.

\begin{tabular}{|c|c|c|c|c|}
\hline \multirow{2}{*}{\multicolumn{2}{|c|}{ Factors }} & \multicolumn{3}{|c|}{ Levels } \\
\hline & & 1 & 2 & 3 \\
\hline Median filter size & $\mathrm{A}$ & 30 & 40 & 60 \\
\hline $\begin{array}{c}\text { Subtraction threshold } \\
\text { value }\end{array}$ & B & 15 & 20 & 25 \\
\hline $\begin{array}{c}\text { Thresholding method } \\
\text { (Binarization) }\end{array}$ & $\mathrm{C}$ & $\begin{array}{c}\text { Global } \\
\text { threshold }\end{array}$ & Otsu & $\begin{array}{l}\text { Valley- } \\
\text { emphasis }\end{array}$ \\
\hline Gaussian filter size & $\mathrm{D}$ & 3 & 5 & 7 \\
\hline $\begin{array}{l}\text { Gaussian filter sig. } \\
\text { (standard deviation of } \\
\text { gaussian curve) }\end{array}$ & F & 0.1 & 0.5 & 0.8 \\
\hline
\end{tabular}

Table 1. Parameters and their levels

\section{CLASSIFICATION OF A CRACK IMAGE USING NEURAL NETWORK}

In this step, the image can be automatically divided with a cracked and non-cracked concrete image using multi-layer artificial neural network (ANN). The required data of ANN are obtained from the processed images in section 2; the data is arranged by the matrix containing features of the each object in each image such as the number of pixels and the ratio of the major axis to minor axis for connected pixels area. When the object has many pixels and high ratio of the major axis to minor axis, there is a strong possibility that it is a crack object.

It is required to distinguish cracks from normal concrete surface automatically to reduce the effort of the human inspectors. In this paper, ANN are adopted to replace the human intelligence, that is to say, to replace the human classification though visual inspection. The proposed algorithm, the multi-layer ANN is basically used and backpropagation algorithm is used for training the NN.

Input layer has 1 layer and consists of 200 neurons. The input data of NN are acquired from processed images; acquired data is sorted by descending order of the number of pixel. This data is provided sequentially into the NN until 200 columns of sorted matrix. That is to say, only the two hundreds of objects are considered in the classification. Hidden layer is composed of 5 layers and each layer has 5 neurons. The number of hidden layer is decided by the experience. Here, too many neurons reduce the calculation 
speed of the network. Output layer is made up of one neuron. Output value represent the classification result, so it could be cracked(value 1) or non-cracked concrete image(value 0). That is decided by the criteria for classification. A log sigmoid function is used in each layer.

$\mathrm{NN}$ is trained by adjusting the weighting factors. As training is processed, output value approached to desired value. After 10000 epochs learning, RMS error is converged into 0.00036 as shown in Fig. 7.

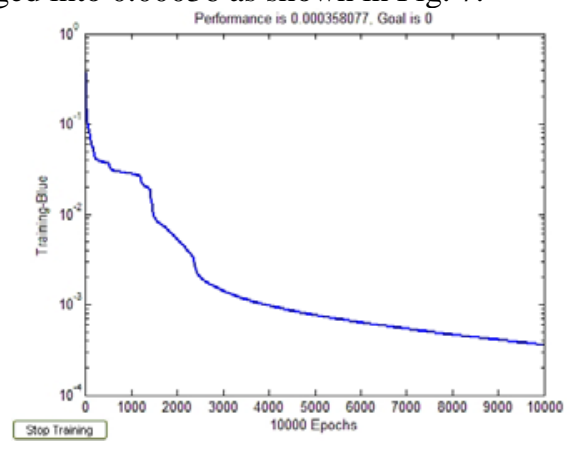

Fig. 7 Training with TRAINGDM

\section{EXPERIMENTS RESULT}

In order to evaluate the usability of the proposed algorithm, we used the concrete surface image of an actual bridge. Images were acquired under the different illumination and background conditions. Image size is $160 \times 120$, and it is composed of RGB color. In the step 1 , total 225 images are processed using the image processing techniques described in section 2 and then features such as object size and its ratio of axis are obtained. The 105 images are used for training the ANN and the 120 images are used for testing. Among 105 images used in training, the number of cracked image is 70 and that of non-cracked image is 35. After training network once with this images, two experiments were performed with different set of test image as shown in Table 2. In this training stage, we need to set the reference output whether the corresponding image contains crack or not. In the image processing step, the required parameters are optimally selected by Taguchi method, and they are $A_{2} B_{1} C_{1} D_{1} F_{1}$ in Table 1 . The optimal parameters determined by Taguchi method affect on the performance of crack visualization as well as the performance of classification. The experimental results on the crack classification are summarized in Table. 2. The recognition rate of the crack image was $90 \%$ and non-crack image was $92 \%$.

\begin{tabular}{|c|c|c|c|c|c|c|c|}
\hline & \multicolumn{2}{|c|}{ Train images } & \multicolumn{2}{|c|}{ Test images } & \multicolumn{3}{|c|}{ Accuracy (\%) } \\
\hline & Crack & $\begin{array}{l}\text { Non- } \\
\text { crack }\end{array}$ & Crack & $\begin{array}{l}\text { Non- } \\
\text { crack }\end{array}$ & Crack & $\begin{array}{l}\text { Non- } \\
\text { crack }\end{array}$ & Total \\
\hline $1^{s t}$ & \multirow[t]{2}{*}{70} & \multirow[t]{2}{*}{35} & 36 & 24 & 92 & 92 & \\
\hline $2^{\text {nd }}$ & & & 36 & 24 & 89 & 92 & \\
\hline & & & & & 90.5 & 92 & 90.25 \\
\hline
\end{tabular}

Table 2. Experiments result

\section{CONCLUSIONS AND FUTURE WORK}

In order to evaluate the safety of a concrete structure, a method to detect cracks from camera image was proposed. First, it was possible to visualize the concrete crack easily through the image processing techniques such as improved subtraction method, filtering and segmentation method. Second, the existence of cracks in many images could be automatically identified using the trained ANN. The proposed algorithm was verified with the real concrete surface image of a bridge and the result shows high accuracy in image classification. However, the test had the limitation that it was performed at the similar environmental conditions such as similar weather, existence of fog, hue of the concrete surface, the shape of structures. It means that construction environment is very diverse and the proposed algorithm needs to be evaluated in various fields of application. The algorithm also needs to be improved for the better accuracy. In addition, the various optimization methods can be applied to determine optimal parameters required in the image processing. It is important in the visualization of crack and the acquisition of the exact crack information.

\section{ACKNOWLEDGEMENT}

This research was supported by Basic Science Research Program through the National Research Foundation of Korea(NRF) funded by the Ministry of Education, Science and Technology(No. 2010-0024807)

\section{REFERENCES}

[1] Chae, M. and Abraham, D., "Neuro-fuzzy approaches 
for sanitary sewer pipeline condition assessment,” Journal of Computing in Civil Engineering, Vol. 15(1), pp. 4, 2001. [2] Khanfar, A., Abu-Khousa, M., and Qaddoumi, N., "Microwave near-field nondestructive detection and characterization of disbonds in concrete structures using fuzzy logic techniques,” Composite Structures, Vol. 62(3), pp. 335-339, 2003.

[3] Adbel-Qader, I., Abudayyeh, O., and Kelly, M., "Analysis of edge-detection techniques for crack identification in bridges," Journal of Computing in Civil Engineering, Vol. 17(4), pp. 255, 2003.

[4] Guo, W., Soibelman, L., and Garrett Jr, J., “Automated defect detection for sewer pipeline inspection and condition assessment," Automation in Construction, Vol. 18(5), pp. 587-596, 2009.

[5] Ng, H., "Automatic thresholding for defect detection," Pattern Recognition Letters, Vol. 27(14), pp. 1644-1649, 2006.

[6] Sinha, S. and Fieguth, P., "Neuro-fuzzy network for the classification of buried pipe defects," Automation in Construction, Vol. 15(1), pp. 73-83, 2006.

[7] Thompson, C., and Shure, L., Image Processing Toolbox: For Use with MATLAB, The MathWorks, 1993.

[8] Fujita, Y., and Hamamoto, Y., "A robust automatic crack detection method from noisy concrete surfaces," Machine Vision and Applications, pp. 1-10, 2010.

[9] Gonzalez, R. and Woods, R., Digital image processing, Prentice Hall, 2002.

[10] Taguchi, G., Taguchi Methods: Research and Development, Quality Engineering Series, Vol. 1, American Supplier Institute, Inc., 1992 